\title{
EXPLORANDO O USO DO MODELO TARGET: A GESTÃO DE LIÇÕES APRENDIDAS NA ÁREA DE PROJETOS EM UMA INSTITUIÇÃO GOVERNAMENTAL DE SEGURANÇA PÚBLICA
}

\author{
EXPLORING THE USE OF THE TARGET MODEL: MANAGEMENT OF LESSONS LEARNED IN THE AREA \\ OF PROJECTS AT A GOVERNMENT PUBLIC SAFETY INSTITUTION
}

EXPLORANDO EL USO DEL MODELO TARGET: LA GESTIÓN DE LECCIONES APRENDIDAS EN EL ÁREA DE PROYECTOS EN UNA INSTITUCIÓN GUBERNAMENTAL DE SEGURIDAD PÚBLICA

JOSE BENEDITO DAMASCENO JR.

Mestre

Universidade Nove de Julho - Brasil damascj@@gmail.com

MARCIRIO SILVEIRA CHAVES

Doutor

Pontifícia Universidade Católica do Rio Grande do Sul - Brasil mschaves@gmail.com

Submetido em: 04/08/2016

Aprovado em: 06/04/2017

Doi: alcance.v24n1.p147-160

\section{RESUMO}

Cada vez mais o tema lições aprendidas em projetos vem se fazendo presente, haja vista a sua abordagem nas instituições e a crescente produção de pesquisas acadêmicas. Modelos para Gestão de Lições Aprendidas (GLA) vêm sendo desenvolvidos, apoiados em processos e ferramentas como os softwares sociais (e.g. Wikis, Blogues e Microblogues). Considerando o problema do desuso ou do desconhecimento de processos e ferramentas para GLA nas instituições, esta pesquisa empírica tem como objetivo validar um modelo de GLA em uma instituição governamental de segurança pública. O modelo foi instanciado em uma plataforma Wiki. Este trabalho usa 0 paradigma de pesquisa Design Science Research (DSR) e o método de pesquisa Technical Action Research (TAR). Os resultados sugerem incrementos ao modelo instanciado na relação processos de lições aprendidas por fase do ciclo de vida do projeto, enquanto a utilidade e a facilidade no uso da Wiki foram reconhecidas pelos envolvidos que, também, consideram haver efeito positivo na eficiência e na eficácia nos processos e nas atividades do projeto. A contribuição para a teoria ocorre à medida que se confirma que investir na adoção de técnicas e ferramentas em projetos é fundamental e que é possível inovar na segurança pública brasileira por meio das boas práticas de projetos. Já a contribuição para a prática se apresenta na adoção de uma sistemática para GLA em projetos inédita naquela instituição.

Palavras-chave: Gestão de Projetos. Gestão de Lições Aprendidas. Projetos do Setor Público. Web 2.0. Wiki.

\section{ABSTRACT}

The theme of lessons learned through projects is becoming increasingly prevalent, given its approach in institutions, and the growing production of academic research on the topic. Models for Management of Lessons Learned (GLA) have been developed, based on processes and tools such as social software (e.g. Wikis, Blogs and Microblogs). Considering the problem of disuse or lack of knowledge of processes and tools for GLA in institutions, this empirical research aims to validate a GLA model in a government public safety institution. The model was placed in a Wiki platform. This work uses the Design Science Research (DSR) paradigm and the method of Technical Action 
Research (TAR). The results suggests increases to the model placed in the list of processes of lessons learned by phase of life cycle of the Project, while the utility and facility in the use of Wiki were recognized by those involved, who also consider that there is a positive effect on the efficiency and efficacy in the processes and activities of the Project. The contribution to theory lies in the confirmation that investing in the adoption of techniques and tools in projects is essential, and that it is possible to innovate in Brazilian public safety through good project practices. The contribution to practice is presented in the adoption of a systematic model for GLA in new projects at that institution.

Keywords: Project Management. Management of Lessons Learned. Public Sector Projects. Web 2.0. Wiki.

\section{RESUMEN}

Cada vez se hace más presente el tema de las lecciones aprendidas en proyectos, considerando su abordaje en las instituciones y la creciente producción de investigaciones académicas. Se están desarrollando Modelos para la Gestión de Lecciones Aprendidas (GLA), apoyados en procesos y herramientas como los softwares sociales (e.g. Wikis, Blogs y Microblogs). Considerando el problema del desuso o del desconocimiento de procesos y herramientas para GLA en las instituciones, esta investigación empírica tiene como objetivo validar un modelo de GLA en una institución gubernamental de seguridad pública. El modelo fue instanciado en una plataforma Wiki. Este trabajo usa el paradigma de investigación Design Science Research (DSR) y el método de investigación Technical Action Research (TAR). Los resultados sugieren incrementos al modelo instanciado en la relación procesos de lecciones aprendidas por fase del ciclo de vida del proyecto, mientras que la utilidad y la facilidad en el uso de la Wiki fueron reconocidas por los involucrados que también consideran que hay un efecto positivo en la eficiencia y en la eficacia en los procesos y en las actividades del proyecto. La contribución para la teoría ocurre en la medida en que se confirma que es fundamental invertir en la adopción de técnicas y herramientas en proyectos y que es posible innovar en la seguridad pública brasileña por medio de las buenas prácticas de proyectos. La contribución para la práctica, en cambio, se presenta en la adopción de una sistematización para GLA en proyectos inédita en aquella institución.

Palabras clave: Gestión de Proyectos. Gestión de Lecciones Aprendidas. Proyectos del Sector Público. Web 2.0. Wiki.

\section{INTRODUÇÃO}

Um olhar crítico sobre as distintas realidades enfrentadas nos projetos é um desafio global para se repensar a gestão de projetos, contribuindo para tornar o Rethinking Project Management (RPM) muito mais difundido e aceito. Seis categorias abrangentes e relacionadas à gestão de projetos surgem: contextualização, aspectos sociais e políticos, necessidade de se repensar a prática, complexidade e incerteza, realidade dos projetos e mais ampla conceituação (SVEJVIG; ANDERSEN, 2014). O repensar da prática, a complexidade e a incerteza em projetos, bem como os aspectos sociais relacionados ao compartilhamento de conhecimento por meio dos softwares sociais, têm pressionado os estudiosos de gestão a pesquisar as regularidades do uso do conhecimento em projetos. Adicionalmente, procura-se compreender os modelos de gestão de projetos e de conhecimento para criar estratégias que capturem e integrem o conhecimento de projetos em toda a organização (LINDNER; WALD, 2011; VIROLAINEN, 2014).

A escolha dos processos e das ferramentas para apoio aos projetos e às lições aprendidas nas organizações necessita considerar a necessidade do cliente de uma forma explícita seus fatores organizacionais e experiências da organização (STANDING; KINITI, 2011; VON KROGH, 2012). Os projetos na área de segurança pública também podem fazer uso das boas práticas de GLA associadas ao uso de ferramentas da Web 2.0. As boas práticas de GLA em projetos de segurança pública agregam valor à gestão do conhecimento e podem ser replicadas em qualquer unidade federativa (BRAGA; CIPRIANO, 2009).

A pesquisa sobre GLA em projetos é fortemente incentivada na literatura devido a uma classe de problemas que incluem a falta de uma estrutura consistente e uma abordagem sistemática para produzir, armazenar e compartilhar lições aprendidas; desuso ou desconhecimento de processos de lições aprendidas em projetos; desuso ou desconhecimento de ferramentas para GLA; falta de local específico em que as lições aprendidas são armazenadas e podem ser acessadas; e uso de planilhas com lições aprendidas que contêm uma grande quantidade de dados históricos difíceis de gerenciar devido ao crescimento incontrolável do conteúdo que 
afeta a sua qualidade e pode levar a erros de interpretação (WIEWIORA; MURPHY, 2013). Diante desta classe de problemas, este artigo investiga a seguinte questão de pesquisa: Em que medida um modelo suportado por uma wiki contribui para o GLA em uma instituição governamental de segurança pública?

\section{REFERENCIAL TEÓRICO}

\subsection{Projeto, o ciclo de vida e o gerenciamento em projetos}

Projeto é um empreendimento temporário feito para criar um produto, serviço ou resultado único (PMI, 2013). Já Kerzner (2011) considera projeto como sendo quaisquer atividades e tarefas que: possuem um objetivo específico a ser atingido dentro de determinadas especificações; possuem datas de início e término definidas; possuem limites de financiamento (se aplicável); consomem recursos humanos e não humanos (dinheiro, pessoas, equipamentos dentre outros) e são multifuncionais (cruzam diversas linhas funcionais).

No que tange ao ciclo de vida em projetos, Verzuh (1999) propõe uma estruturação dos projetos por meio dos processos: definição, planejamento, execução e encerramento. Estes processos se repetem ao longo dos vários estágios do ciclo de vida do projeto (ver Figura 1). Maximiano (2002) também compartilha deste pensamento ao afirmar que os processos da administração de projetos - planejamento, organização, execução e controle são necessários para todos o projeto e para cada fase do seu ciclo de vida. O PMI (2013), por sua vez, propõe 47 processos para o gerenciamento de projetos, agrupados em cinco grupos de processos. Esses cinco grupos de processos são: Iniciação, Planejamento, Execução, Monitoramento e Controle e Encerramento.

Figura 1: Sequência típica de fases no ciclo de vida de um projeto

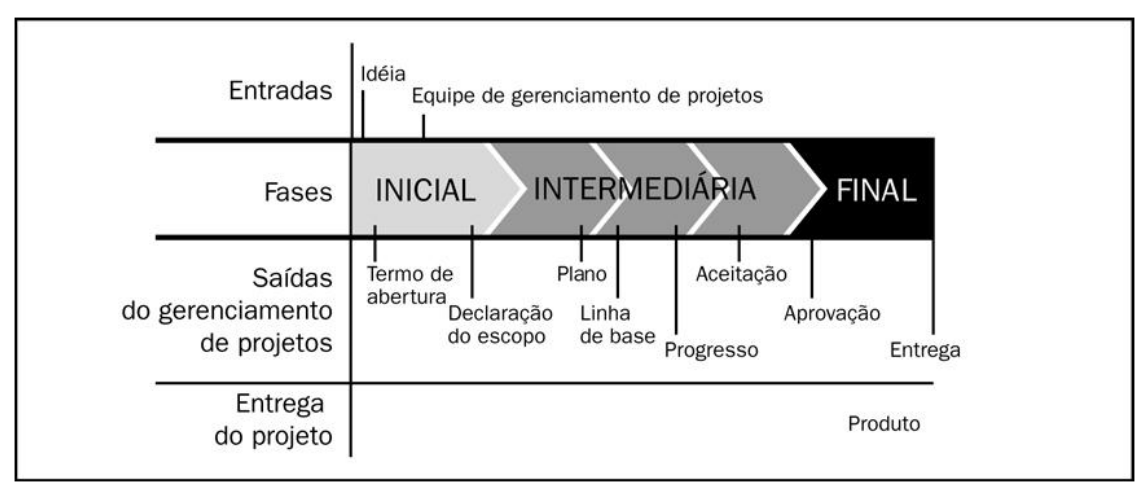

Fonte: PMI, 2013

Enquanto que o gerenciamento de projetos é, segundo Kerzner (2011), o planejamento, a organização, a direção e o controle dos recursos da empresa para um objetivo de relativo curto prazo, que foi estabelecido para concluir metas e objetivos específicos. O PMI (2013) define o gerenciamento de projetos como a aplicação de conhecimento, habilidades, ferramentas e técnicas às atividades do projeto para atender aos seus requisitos.

A abordagem de gerenciamento de projetos é caracterizada por métodos de reestruturação da administração e da adaptação de técnicas especiais de gestão, com o objetivo de obter melhor controle e utilização dos recursos existentes (KERZNER, 2011). Carvalho \& Rabechini (2011) sugerem que investir na adoção de técnicas e ferramentas de gerenciamento de projetos é fundamental, o que tem sido uma preocupação crescente nas empresas.

\subsection{Projetos na Área de Segurança Pública}

Muitos são os aspectos que recomendam observar a segurança pública brasileira com ferramentas de acompanhamento de longo prazo. Evidencia-se que, no cenário que está posto para a segurança pública do país, a gestão de projetos complexos - característica do setor público - é uma possibilidade que se apresenta como meio de conciliação entre duas vertentes centrais de análise - a complexidade e a incerteza - que são reciprocamente associáveis. É possível inovar na segurança pública brasileira por meio da gestão de projetos, de 
modo que redes de órgãos públicos integradas nos entes federativos sejam coordenadas em busca de objetivos desafiadores de longo prazo (REIS, 2009).

Como protagonista no atual palco de gestão em segurança pública que se estabelece no Brasil, a adoção da metodologia de gerenciamento de projetos possibilita, à ação local, regional e federal, a superação de inúmeros obstáculos à efetividade dos resultados dos seus projetos, tais como: objetivos não realistas; falta de controle das mudanças; complexidade do projeto; prazos não cumpridos ou não realistas; parceiros que não cumprem sua parte; surgimento de imprevistos; lentidão nas aquisições; falta de participação do cidadão, entre outros empecilhos que contribuem para a não ocorrência de resultados duradouros e sustentáveis ao longo do tempo (BRAGA; CIPRIANO, 2009).

As principais diferenças observadas no gerenciamento de projetos do setor público em relação ao privado são as seguintes (OLIVEIRA FILHO, 2012): a. Critérios de seleção: grande parte dos projetos de governo é iniciada principalmente para atender a necessidades das áreas de saúde, segurança, bem-estar, socioeconômicas e ambientais com viés predominantemente social; $b$. São pautados pelo ciclo anual do orçamento, o que constitui uma restrição significativa no gerenciamento de projetos de governo. Os orçamentos são tipicamente alocados para um ano fiscal, obrigando a divisão de projetos e programas em módulos de um ano. c. Sofrem grande influência do ciclo eleitoral, não só pela possibilidade de reversão de políticas em caso de mudança da administração, como por restrições legais estabelecidas pelo princípio da neutralidade em período eleitoral; $d$. São objeto de regulamentação intensa e pesada nas aquisições do governo, pela necessidade de assegurar igualdade de oportunidade para os licitantes de obras e serviços públicos, bem como probidade por parte do órgão adquirente. $O$ gerente de projetos do setor público deve estar, portanto, plenamente a par de tais peculiaridades, de forma a garantir o êxito do seu projeto (OLIVEIRA FILHO, 2012).

\subsection{Gestão de Lições Aprendidas}

Os seres humanos, que são quase os únicos a terem a capacidade de aprender com a experiência dos outros, também são notáveis por sua falta de inclinação evidente para fazê-lo (ADAMS, 1995). Uma pesquisa com mais de 180 respondentes, dentre eles especialistas em gestão de projetos, aponta que quanto ao uso ou à realização de GLA, $47 \%$ dos respondentes indicaram que, sempre ou frequentemente, realizam a GLA nos seus projetos; $42,5 \%$ informaram que raramente ou nunca realizam a GLA e 10,5\% afirmaram desconhecer se há algum processo de GLA em andamento na organização (ROSA et al., 2016). Considera-se, portanto, que a GLA é ainda um tema desafiador.

A ideia de GLA em uma organização é que, através de uma abordagem formal para a aprendizagem, os indivíduos e a organização podem reduzir o risco de repetição de erros e aumentar a chance de que os êxitos são repetidos. Lições aprendidas descrevem mais do que aprender com a experiência. A aprendizagem deve ser usada para justificar mudanças que levem a um melhor desempenho (NATO, 2011).

As lições aprendidas são ativos intelectuais usados para criar valor com base na experiência do passado. Da mesma forma, as lições aprendidas contribuem para uma agenda de aprendizagem organizacional (CARRILLO et al.,2013). Os autores ainda citam que a gestão do conhecimento é a identificação, otimização e gestão ativa dos ativos intelectuais para criar valor, aumentar a produtividade e suportar a vantagem competitiva.

A retenção sistemática de experiências de projetos permite que uma empresa possa comparar seus diversos projetos mais sistematicamente e documentar seus problemas com mecanismos mais eficazes para resolução (SCHINDLER \& EPPLER, 2003). Experiências que são, por definição, vinculadas a pessoas que estão pessoalmente envolvidas na resolução de problemas, muitas vezes não são processos de uma parte de uma documentação do projeto e eles raramente são transferidos para outras pessoas durante o curso de um projeto (SCHINDLER \& EPPLER, 2003).

O processo de lições aprendidas pode ser decomposto em cinco subprocessos (WEBER et al., 2001):

- Coletar: envolve coletar as lições aprendidas;

- Verificar: time de especialistas realiza este subprocesso para validar as lições aprendidas coletadas, avaliando redundância, consistência e relevância;

- Armazenar: abrange itens da abstração das lições aprendidas e endereça formatação, escolha e arquitetura do repositório, indexação. As lições podem estar de forma estruturada, semiestruturada e também podem ser mantidas em diferentes tipos de mídia; 
- Disseminar: a disseminação é tida como um dos mais importantes subprocessos, já que promovem a lição a ponto de ser possivelmente reutilizada;

- Reutilizar: a escolha de reutilização da lição ou não é do usuário, pois uma reutilização automática depende de uma arquitetura de lição aprendida embutida no sistema, o que é raro.

Normalmente, metodologias e processos requerem suporte por meio de ferramentas para que possam assim potencializar a sua utilização. As ferramentas Web 2.0 podem se apresentar como um facilitador neste sentido (CHAVES et al., 2016a, VERONESE \& CHAVES, 2016).

\subsection{Ferramentas Web 2.0}

O termo Web 2.0 foi oficialmente adotado pela empresa O'Reilly Media em 2004. Ele se refere à segunda geração dos serviços Web baseados na comunidade como site de redes sociais, blogues, microblogues, wikis, entre outras, a qual promove uma Web mais socialmente conectada, em que todos sejam capazes de comunicarse, participar, colaborar e incluir e editar o espaço de informações (PACHLER \& DALY, 2009). A Web 2.0 revolucionou como as pessoas compartilham informações e conhecimento. As ferramentas da Web 2.0 passaram por um incrível crescimento nos últimos anos. Aplicativos como Wikipedia, Facebook, Youtube, Twitter e Linkedin foram muito bem-sucedidos em formar ambientes participativos (STOCKER \& MÜLLER, 2013).

Por outro lado, o uso de ferramentas sociais ainda é um desafio. Estudos (GRACE, 2009; MAJCHRZAK; WAGNER \& YATES, 2006) destacam algumas motivações que podem justificar a não inclusão das ferramentas sociais nas empresas e em projetos, como a falta de cultura dos colaboradores na aplicação de tecnologias 2.0 nas empresas.

A aceitação do uso de tecnologias Web 2.0 em ambiente corporativo pode ser analisada sobre diversos aspectos que influenciam a intensão de compartilhamento do conhecimento por indivíduos (PAPADOPOULOS; STAMATI \& NOPPARUCH, 2013; GHOLAMI \& MURUGESAN, 2011; VON KROGH, 2012), a exemplo da teoria social cognitiva, cuja imagem é a recompensa. A teoria social cognitiva associa a confiança das pessoas em partilhar com as recompensas resultantes. A recompensa esperada por indivíduos vem em forma de benefícios como reputação e relacionamento esperado.

A crescente adoção de equipes virtuais em projetos impulsionados pela globalização colabora com a necessidade da mobilidade dos funcionários e da necessidade de tomada de decisão coletiva e rápida por membros que estão em locais diferentes. Isto contribui para o uso das ferramentas colaborativas da Web 2.0 - as wikis, os blogues, os microblogues, os fóruns de discussão e as plataformas de redes sociais - no suporte à tomada de decisão (TURBAN; LIANG \& WU, 2011). 0 uso de Wikis para apoiar a disseminação e a aplicação de lições aprendidas em projetos é sugerido por Duffield \& Whitty (2015) e implementado em Chaves, Tessi, Winter \& Damasceno (2016). As Wikis são as ferramentas Web 2.0 que mais são indicadas e recomendadas em todos os processos de lições aprendidas (ROSA et al., 2016).

De um ponto de vista mais organizacional, Wiki é uma simples, mas poderosa ferramenta colaboradora baseada em um sistema da Web ou, até mesmo, de gestão de conteúdo para criação e edição do material a ser armazenado (GHOLAMI \& MURUGESAN, 2011). A Wiki tem sido descrita como uma ferramenta de democratização do conhecimento (MELOCHE et al., 2009).

As tecnologias de mídia social estão fazendo incursões rápidas em empresas (RIEMER \& SCIFLEET, 2012). No contexto do trabalho de conhecimento intensivo, as proposições de melhoria da comunicação, compartilhamento de informações e envolvimento do usuário parecem particularmente promissoras, pois os resultados apresentados com o uso de tecnologias sociais estão cada vez mais agregando valor nos produtos e nos serviços (RIEMER \& SCIFLEET, 2012). Alguns grupos usam plataformas Wiki para facilitar a gestão de projetos (BEBENSEE et al., 2012; WINTER \& CHAVES, 2017). Informações e experiências registradas em relatórios de projetos e manuais de como foram executados os projetos anteriores são recuperadas a partir da plataforma Web, principalmente para facilitar a comunicação e a colaboração entre os membros de equipes de projeto (CHAVES et al., 2016b).

Por meio de Wikis, as informações podem ser consultadas a qualquer momento e os membros da equipe corrigir os problemas ali mencionados em tempo real (STOCKER et al., 2012). Isso colaboraria diretamente com as lições aprendidas, gerando uma consciência mais efetiva do potencial destas ferramentas, contribuindo diretamente sobre o poder estratégico que pode ser agregado em diversos projetos, reduzindo custos e tempo.

Revista Alcance - Eletrônica - vol. 24 - n. 1 - jan./mar. 2017 
A falta de diretrizes claras no uso Wiki, porém, principalmente onde as empresas não possuem políticas definidas, pode levar a confusões (STANDING \& KINITI, 2011). Os autores destacam, também, os principais fatores que devem ser considerados pelas empresas num processo de adoção de Wikis: (1) propósito claro para a criação da Wiki; (2) ambiente com uma cultura de colaboração desenvolvido; (3) capacidade de gestão do conhecimento; (4) processo formal de inovação na empresa; e (5) gestão clara de intenção e propósitos. Wikis requerem um objetivo para o seu uso. Identificou-se que algumas Wikis foram adotadas apenas com objetivos vagos e isso muitas vezes resulta em uma falta de participação dos colaboradores.

0 uso de processos de lições aprendidas associado ao ciclo de vida do projeto e à utilização de ferramentas Web 2.0 é retratado no modelo proposto por Rosa et al. (2016) para a GLA em projetos (Figura 2).

\subsection{0 modelo instanciado: Target}

Rosa et al. (2016) propõem um modelo para suportar a GLA em projetos baseados em ferramentas da Web 2.0, conforme a Figura 2, que representa o modelo Target.

Figura 1: Estrutura do modelo Target.

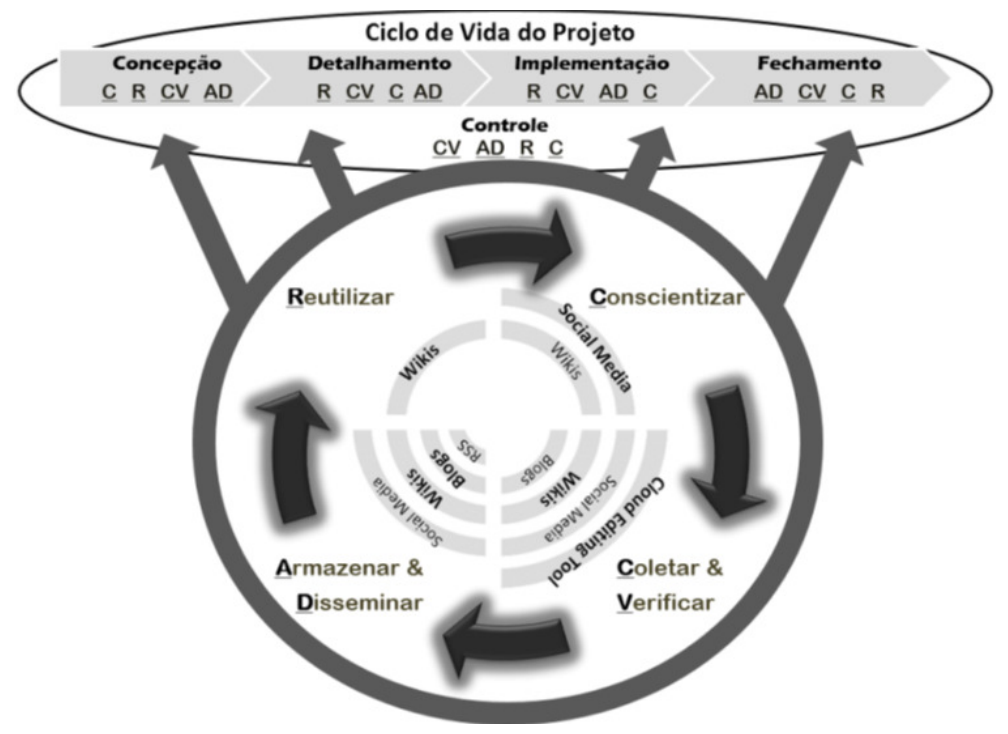

Fonte: Rosa et al. (2016, p. 104)

A parte superior do Target apresenta as fases do ciclo de vida do projeto. Para cada fase, podem ser utilizados os processos de lições aprendidas, e abaixo de cada fase é evidenciada a ordem de priorização de cada processo. A ordem de priorização sugere quais processos seriam mais indicados em determinada fase, em ordem de importância para sua aplicação. Quanto à gestão de projetos, o Target apresenta os processos de forma que estes possam ser utilizados em qualquer fase do ciclo de vida do projeto, evitando que a GLA seja inflexível e sugerindo que os diversos processos possam acontecer em qualquer momento do projeto (ROSA et al., 2016).

Rosa et al. (2016) comentam que, das fases do ciclo de vida do projeto, ilustra-se o relacionamento dos processos de lições aprendidas com as ferramentas da Web 2.0. Para cada processo foi selecionada uma ferramenta específica. A ferramenta Wiki apresenta-se comum a todos os processos e fases do ciclo de vida do projeto. A Wiki é reconhecida como o repositório oficial das informações de lições aprendidas. Os blogues e as redes sociais são úteis para fomentar a discussão. 0 Office na Web é uma ferramenta útil para montar um repositório de informações temporárias para os indivíduos ou para um projeto, de forma que todos possam colaborar no mesmo documento, até que este passe ao repositório oficial. A RSS é a ferramenta para disseminar as informações e que pode estar presente ou embutida na plataforma Wiki (ROSA et al., 2016).

\subsection{Desenho da pesquisa}


Objetivando atender às necessidades do usuário em seu ambiente no que tange à GLA em projetos por meio da validação do modelo Target, este trabalho adota o paradigma de pesquisa Design Science Research DSR e o método Technical Action Research - TAR. Hevner (2007) apresenta uma visão do Design Science Research fundamentado em três ciclos, conforme ilustra a Figura 3:

Figura 3: Ciclos do Design Science Research

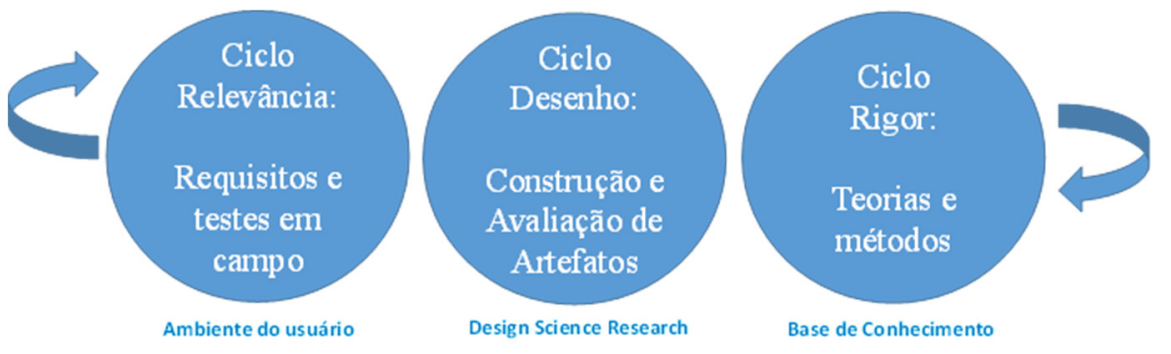

Fonte: Adaptado de Hevner (2007).

O ciclo de relevância inicia com uma aplicação que não só fornece os requisitos para a pesquisa (por exemplo, a oportunidade ou problema a ser abordado) como entradas, e também define critérios de aceitação para a avaliação final dos resultados da investigação. É relevante para o usuário assegurar que 0 artefato pode melhorar o seu ambiente e como isso poderá ser medido, bem como se o estudo de campo do artefato pode ser executado por meio de métodos de transferência de tecnologia apropriada (HEVNER, 2007). 0 ciclo de desenho do artefato considera a construção de um artefato, a sua avaliação e o feedback posterior para refiná-lo ainda mais. Durante a execução do ciclo de criação, é importante manter um equilíbrio entre os esforços despendidos na construção e na avaliação da evolução do artefato desenhado. Ambas as atividades devem ser convincentes e baseadas em relevância e rigor. Havendo um argumento forte para a construção do artefato, é insuficiente se a avaliação subsequente for fraca. Já em relação ao ciclo de rigor, Hevner (2007) explica que este fornece conhecimento passado para o projeto de pesquisa para assegurar a sua inovação. Depende dos pesquisadores uma pesquisa aprofundada que referencie a base de conhecimento, a fim de garantir que os desenhos produzidos sejam contribuições da pesquisa. Rigor na pesquisa baseia-se na seleção e na aplicação das teorias e dos métodos adequados para a construção e avaliação do artefato. Adições à base de conhecimento como resultados de pesquisa devem incluir todas as extensões para as teorias e métodos originais feitos durante a pesquisa, novos meta-artefatos (produtos de design e processos) e experiências adquiridas com a realização da pesquisa de campo e teste do artefato no ambiente de aplicação.

Em linha com o paradigma de pesquisa DSR, o método de pesquisa adotado neste trabalho é o TAR Technical Action Research. Enquanto a maioria dos métodos de pesquisa empírica estudam o fenômeno como ele é atualmente, os pesquisadores que usam os métodos de ação (e.g. pesquisa-ação) buscam intervir nas situações estudadas (WIERINGA \& MORALI, 2012). 0 método de pesquisa TAR (Figura 4) ou pesquisa-ação técnica apoia a busca da solução de um problema utilizando um artefato ou prescrição, estreitando a distância entre a teoria e a prática (WIERINGA \& MORALI, 2012). 
Figura 4: Estrutura TAR - Technical Action Research ou Pesquisa-ação Técnica

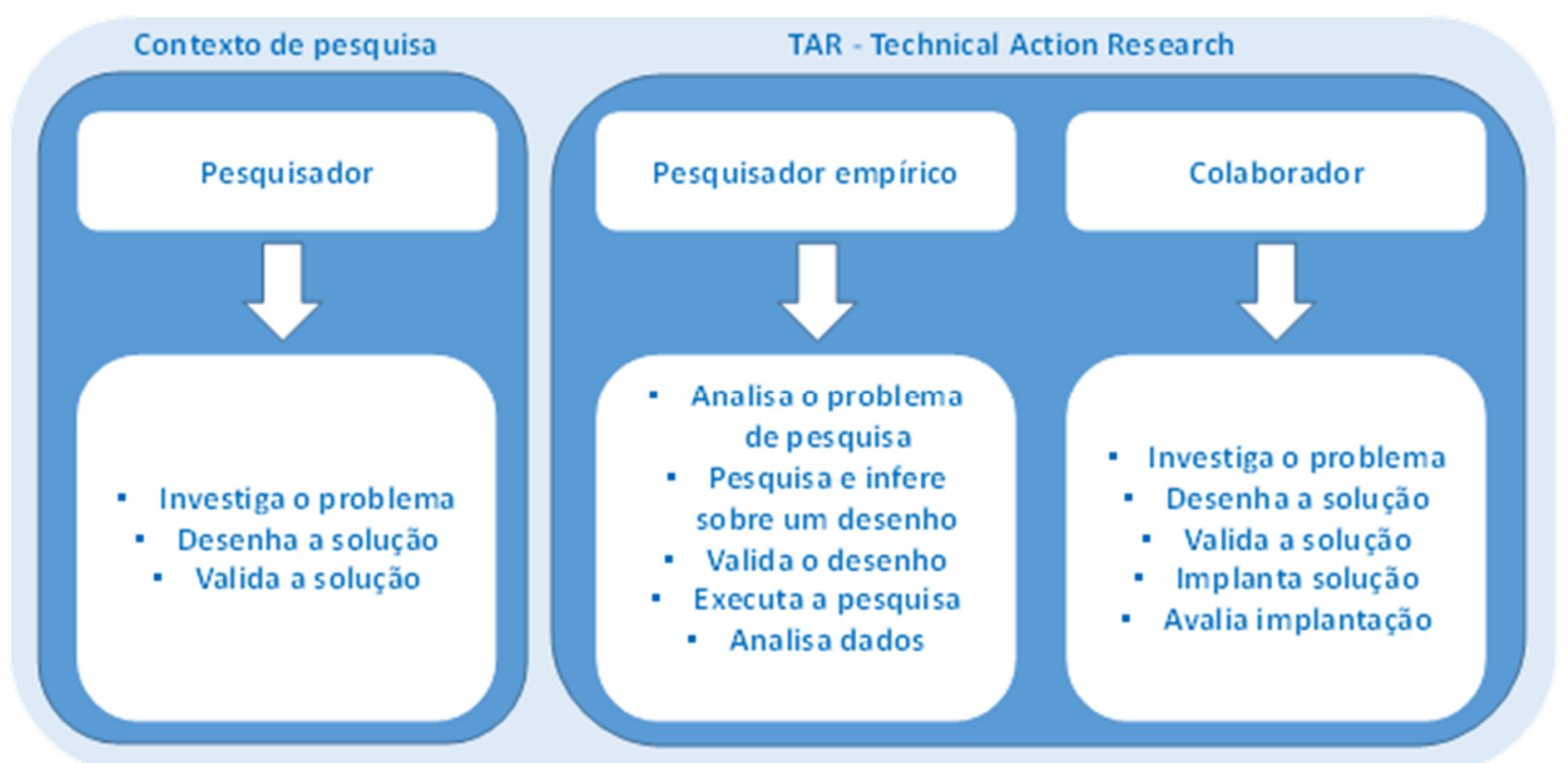

Fonte: Adaptado de Wieringa \& Moralı (2012).

A escolha do método TAR para este estudo insere o uso do modelo Target que, doravante, assume a classificação de artefato Target dentro do contexto da pesquisa: a perspectiva prática que a ciência requer empiricamente (DRESCH, 2014). Este trabalho verifica o grau de utilidade e facilidade (YOON \& KIM, 2007) no uso da ferramenta Wiki; a incidência dos processos de lições aprendidas nas fases do ciclo de vida do projeto (ROSA et al., 2016); e o efeito no desempenho e na qualidade nos processos e nas atividades (GOMES, 2012; MELLO, 2014) do projeto em função da instanciação do artefato Target.

O artefato Target foi validado na área de projetos de uma instituição governamental de segurança pública. Essa área de projetos é constituída por profissionais com formação superior e com experiência em gestão de projetos que atuam, em sua maioria, há mais de 5 anos na área. A Tabela 1 apresenta a qualificação dos entrevistados. Em quase sua totalidade a área é composta por Oficiais (Tenentes, Capitães, Majores e um Tenente-Coronel que é responsável pela área de projetos), mas também há Praças (Cabos e soldados), que promovem a gestão de projetos com a mesma qualificação.

Responsável por prestar policiamento nas modalidades ostensiva e especializada. Essa instituição caracteriza-se pelo desenvolvimento de projetos complexos e multidisciplinares e que, portanto, apresentam riscos na sua execução. Uma base de conhecimento histórica representada por lições aprendidas em projetos vem colaborar para a mitigação desses riscos e um melhor desempenho no desenvolvimento desses projetos.

Tabela 1: Qualificação dos entrevistados

\begin{tabular}{|c|c|l|}
\hline ID Entrevistado & Patente & Grau de Instrução \\
\hline 1 & Tenente & Mestre \\
\hline 2 & Sargento & Graduado \\
\hline 3 & Sargento & Especialista (Pós-Graduação) \\
\hline 4 & Capitão & MBA \\
\hline 5 & Cabo & Especialista (Pós-Graduação) \\
\hline 6 & Sargento & Especialista (Pós-Graduação) \\
\hline 7 & Cabo & Especialista (Pós-Graduação) \\
\hline 8 & Major & Especialista (Pós-Graduação) \\
\hline 9 & Soldado & Graduado \\
\hline
\end{tabular}

Fonte: Dados da pesquisa 
Os dados e as informações do objeto de análise foram coletados por meio de observação direta no ambiente do usuário, da realização de entrevistas com perguntas abertas a cada envolvido e, ainda, com a realização de um grupo focal confirmatório para ratificar ou não os resultados obtidos. A Figura 5 apresenta a dinâmica adotada durante os trabalhos em campo.

Figura 5: Atividades realizadas ao longo do trabalho de campo

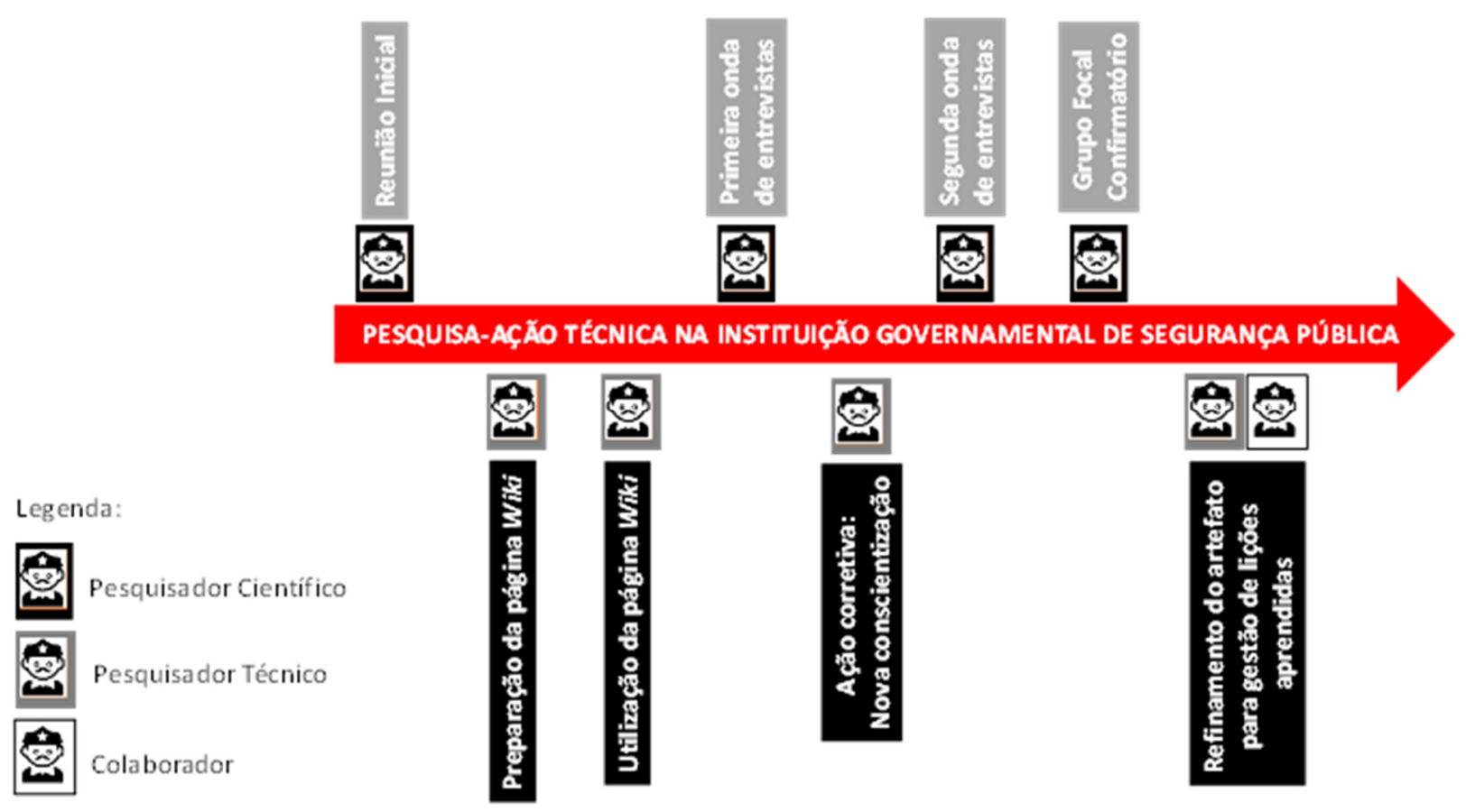

Fonte: Autor.

\section{ANÁLISE DOS RESULTADOS}

Após decorridos 15 dias de utilização da página Wiki desenvolvida exclusivamente para o projeto Alpha, houve a realização de uma primeira onda de entrevistas a quatro integrantes da equipe do projeto, entre oficiais e praças, com a aplicação de 12 perguntas abertas. Essa fase tinha como objetivo verificar a utilidade e a facilidade do uso da ferramenta, a incidência dos processos de lições aprendidas nas fases do ciclo de vida do projeto e, ainda, a eficácia nas tarefas do projeto em função do uso da página Wiki desenvolvida. Já foi possível realizar análises preliminares dessa primeira onda de entrevistas que culminaram numa primeira ação corretiva. Quase todos os entrevistados citaram a importância do processo de lição aprendida: Conscientização.

A conscientização envolve a formalização da importância da lição aprendida, de forma a motivar e incentivar os membros da equipe a realizar as atividades de lições aprendidas nos projetos. Os primeiros entrevistados sugeriram que muito mais lições aprendidas já poderiam estar registradas naquele instante se houvesse uma maior e melhor conscientização das demais partes interessadas no projeto.

Considerando esse primeiro resultado obtido, uma nova divulgação mais clara e objetiva foi realizada, por meio de um novo e-mail do gestor do projeto aos demais envolvidos. O resultado dessa ação corretiva já pode ser aferido à época da segunda onda de entrevistas, na qual mais cinco entrevistados foram abordados. Um número maior de lições aprendidas registradas na página Wiki do projeto já pode ser observado.

Com a finalização da segunda onda de entrevistas individuais, perfazendo um total de 9 entrevistados entre oficiais e praças, foi analisada cada uma dessas entrevistas, com o objetivo de pontuar as impressões de cada entrevistado. Como resultante, foram elaboradas 9 assertivas aplicadas a um grupo de 6 entrevistados, por meio da dinâmica de grupo focal confirmatório.

Quanto à utilidade e à facilidade (YOON \& KIM, 2007) do uso da Wiki na GLA, os entrevistados do grupo focal não foram unânimes. $O$ entrevistado 2 iniciou a dinâmica sinalizando: "A ferramenta requer conhecimento para sua utilização... e nosso público, nem todos, tem esse conhecimento para poder utilizar... talvez, se fosse em 
papel e caneta, muitos poderiam contribuir muito mais". Já o entrevistado 5 , baseado na cultura organizacional da instituição governamental de segurança pública, acrescentou:

Que para a nossa instituição, teríamos mais participações se tivéssemos trabalhado com Fóruns e não com Wiki. A Wiki...as pessoas nem sabem o que é.... depende muito de comunicação, de explicação, de conscientização. Entendo que dentre as possibilidades de gestão participativa, de lição aprendida, hoje a Wiki não é a melhor para a nossa cultura, não foi em 2015, mas, pode ser que agora passe a ser. Tínhamos melhores opções, que a gente acabou não usando que seria o "Fórum", na mesma plataforma (IBM) e é muito mais simples, é só apertar um botão, que já faz a contribuição e não corro o risco de um apagar a opinião do outro. (Entrevistado 5).

No que tange à incidência dos processos de lições aprendidas nas fases do ciclo de vida do projeto (ROSA et al., 2016), a maioria dos entrevistados considerou que "Conscientizar sobre lições aprendidas na fase de iniciação; Registrar lições aprendidas durante a fase de execução; Fazer uso de lições aprendidas durante a fase de planejamento são mais importantes! "

Por fim, em relação à eficácia (GOMES, 2012; MELLO, 2014), todos os entrevistados concordaram que a Wiki produziu um efeito positivo na execução das tarefas do projeto. 0 entrevistado 1 exclamou: "Não tem como não melhorar o desempenho estando bem informado por meio da contribuição alheia" e "Observar erros e acertos alheios permite melhorar o seu trabalho. Por sua vez, o entrevistado 6 indicou: "Sim. Otimiza tempo e recursos" e "É a aplicação da lição aprendida que remete à qualidade". Finalmente, o entrevistado 8 concluiu: "Se não houvesse a Wiki não conseguiríamos facilmente coletar tudo o que foi coletado".

\subsection{Artefato prescrito}

A Figura 6 apresenta o artefato Target prescrito para a GLA em projetos numa instituição governamental de segurança pública. $O$ artefato se apresenta no sentido horário e é resultante de observação em campo, de entrevistas individuais e da realização de um grupo focal confirmatório.

Figura 6: Modelo prescrito para a instituição governamental de segurança pública

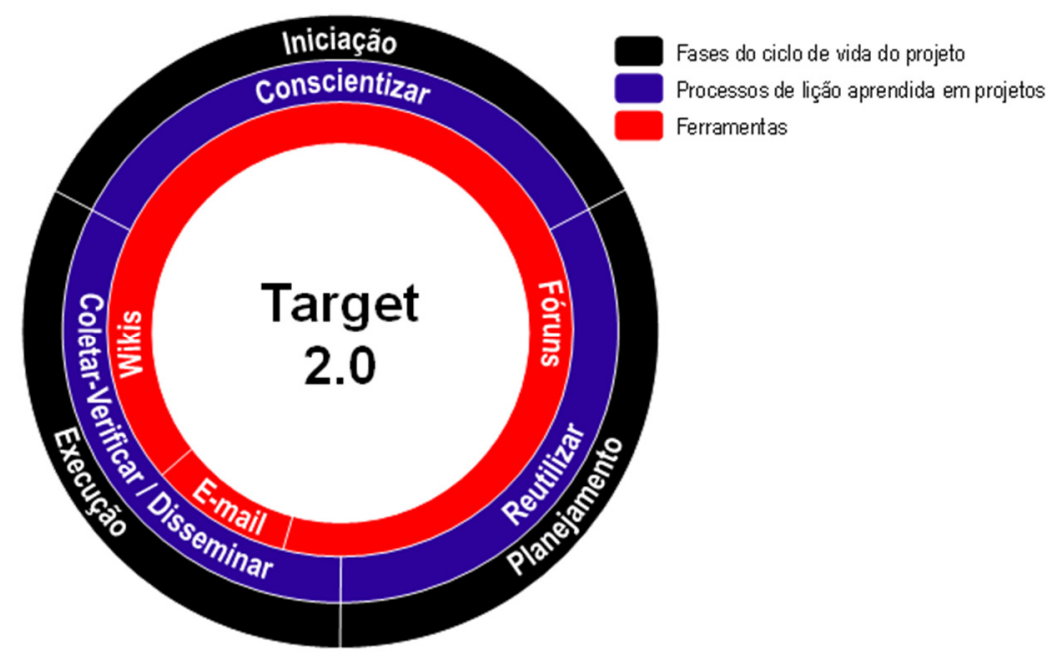

Fonte: Dados da pesquisa.

O primeiro processo de lição aprendida, conscientizar, é executado na fase de iniciação do ciclo de vida do projeto. Este processo tem o principal objetivo de dar ciência e convencer as partes interessadas no projeto sobre a importância e a necessidade de promover a boa prática de lições aprendidas na instituição. Tanto na página Wiki, bem como no caso da adoção de Fóruns para a prática de lições aprendidas, deverá haver uma 
mensagem explicativa seguida de uma ilustração com o objetivo de convidar os usuários dessas ferramentas a refletir sobre o tema. Além do recurso introdutório nessas ferramentas, o gerente de projetos também se utilizará do recurso de e-mail às partes interessadas para inserir o tema lições aprendidas e a ferramenta utilizada no contexto do projeto.

O segundo processo de lição aprendida, reutilizar, é executado na fase de planejamento do ciclo de vida do projeto. Este processo tem como objetivo principal promover o uso de lições aprendidas previamente coletadas por outros colegas em outros projetos. 0 processo de reutilizar contribuirá para potencializar as chances de sucesso no projeto, por meio de um planejamento mais assertivo, que considera experiências anteriores de outrem, erros e acertos em projetos ou situações semelhantes. Normalmente, este processo (reutilizar) é apresentado na literatura, em ordem, como o último processo. Todavia, na instituição governamental de segurança pública, este se apresenta como o segundo processo de três, caracterizando o ciclo a ser praticado em todos os projetos naquela instituição.

O terceiro processo de lição aprendida, coletar/verificar, é executado na fase de execução do ciclo de vida do projeto. Tem como objetivo promover a coleta de lições aprendidas no projeto corrente por parte das partes interessadas, seguida da verificação do teor e do conteúdo coletado por parte do gerente de projetos ou a quem este designar a atividade de verificação. Segundo a instituição governamental de segurança pública, é na fase de execução que tudo acontece e, portanto, devendo ocorrer a grande parte das coletas de lições aprendidas no projeto, assim que elas ocorrem.

O quarto processo de lição aprendida, disseminar, é executado automaticamente pela Wiki, quando uma lição aprendida é coletada ou editada. Um e-mail automático é disparado para o grupo de usuários desta Wiki, o que caracteriza uma disseminação ativa. Também será praticada a disseminação passiva, forma na qual as partes interessadas buscam o conhecimento na ferramenta Wiki ou Fóruns, esta última mais enraizada na corporação.

Durante as entrevistas, não foi mencionado pelos entrevistados o processo de armazenamento. Por inferência, isso pode ter ocorrido pelo fato de a Wiki já possuir uma estrutura própria e pronta para o uso, sem que os usuários da instituição necessitem criá-la ou adequá-la. Também não foram mencionadas pelos entrevistados nas entrevistas individuais e nem citadas no grupo focal confirmatório as fases de monitoramento e controle, bem como a de encerramento do projeto.

Infere-se ainda que as fases de "monitoramento e controle" não foram citadas no desenvolvimento dos trabalhos, uma vez que as mudanças provenientes e aprovadas nestas fases são naturalmente realizadas na fase de "execução" em que "tudo acontece", segundo os entrevistados.

Por fim, em relação à fase de encerramento do projeto, durante uma entrevista individual, foi citado apenas que há um formulário em MS-Word que tem como objetivo o encerramento do projeto. Todavia, este formulário não tem um campo específico e qualificado para o registro de lições aprendidas no projeto.

A área de projetos da instituição governamental de segurança pública em que foi desenvolvida essa pesquisa-ação técnica (TAR) sinalizou que estão sendo revistos os processos para gestão de projetos que, atualmente, ainda estão baseados no guia de boas práticas PMBoK $3^{\mathrm{a}}$ Edição. Com isto, muito provavelmente, poderá ocorrer a revisão do artefato prescrito anteriormente, considerando os processos e/ou as fases do ciclo de vida que ora não foram considerados.

\section{CONTRIBUIÇÕES TEÓRICAS E PRÁTICAS}

Investir na adoção de técnicas e ferramentas de gerenciamento de projetos é fundamental e tem sido uma preocupação crescente nas instituições (CARVALHO \& RABECHINI, 2011). Durante os trabalhos de campo desta pesquisa foi possível comprovar a preocupação da liderança do projeto Alpha em praticar lições aprendidas de maneira sistemática, com o uso de processos e de ferramenta adequados.

Quanto à inovação na segurança pública brasileira por meio da gestão de projetos (REIS, 2009), o uso do Target no projeto Alpha proporcionou uma nova perpectiva para a GLA em projetos, promovendo uma inovação, pois a instituição passou a ter uma sistemática para contribuir para gestão do conhecimento em projetos.

No que se refere ao alinhamento das pessoas na GLA (DUFFIELD \& WHITTY, 2015), constatou-se que muitos dos entrevistados sugeriram a conscientização dos envolvidos no projeto sobre a prática de lições aprendidas como fundamental para o registro de experiências passadas em projetos. 
Em relação à prática, a liderança da área de projetos na instituição pesquisada indicou que, anteriormente ao uso da Wiki na GLA, as informações sobre projetos chegavam a ele "filtradas". Isto se deve provavelmente à hierarquização daquela instituição, podendo as informações terem seu conteúdo e teor alterados, já que passavam por inúmeros interlocutores distintos. Com 0 uso da Wiki, tais informações são prontamente coletadas e disseminadas pela ferramenta entre os envolvidos e, portanto, têm seu conteúdo e teor preservados.

No que diz respeito a contribuições para a área de administração da instituição governamental de segurança pública, para a área de gestão de projetos e para os seus gerentes de projetos, este trabalho ratifica a importância das boas práticas de lições aprendidas de forma sistematizada. A GLA, além de proporcionar o reaproveitamento de conhecimento nos projetos similares (PEMSEL \& WIEWIORA, 2013), pode também contribuir para o conhecimento organizacional em instituições de outros estados da federação.

Por fim, em relação às contribuições para projetos de segurança pública, este trabalho apresenta uma nova forma para tratar com lições aprendidas em projetos neste segmento. Por meio da colaboração social do aprendizado, verificou-se que tanto a complexidade como a incerteza, associadas à falta de conhecimento, podem ser minimizadas.

\section{CONSIDERAÇÕES FINAIS}

Este trabalho validou uma instância do modelo Target para GLA durante um projeto em uma instituição governamental de segurança pública. Por meio do método TAR, foi possível obter uma nova perspectiva desse modelo, gerando um artefato que contribuiu para a GLA em projetos numa instituição que desenvolve projetos complexos e multidisciplinares. Os processos de lições aprendidas foram mais bem compreendidos e uma nova ferramenta, a Wiki, passou a ser reconhecida como um meio eficaz de compartilhamento do conhecimento.

\section{REFERÊNCIAS}

ANDRADE, J.; ARES, J.; GARCÍA, R.; PAZOS, J.; RODRÍGUEZ, S.; RODRÍGUEZ-PATÓN, A.; SILVA, A. Towards a lessons learned system for critical software. Reliability Engineering \& System Safety, v. 92, n. 7, p. 902-913, 2007.

BEBENSEE, T.; HELMS, R.; SPRUIT, M. Exploring Web 2.0 applications as a mean of bolstering up knowledge management. Leading issues in social knowledge management, v. 1, p. 22-45, 2012.

BRAGA, W.; CIPRIANO, N. O gerenciamento de projetos na segurança pública: uma abordagem pragmática da aplicação do método na gestão das ações integradas a partir de um estudo de caso. Revista Brasileira de Segurança Pública, p. $114-132,2009$.

CARRILLO, P.; RUIKAR, K.; FULLER, P. When will we learn? Improving lessons learned practice in construction. International Journal of Project Management, v. 31, n. 4, p. 567-578, 2013.

CARVALHO, M. M.; RABECHINI JR, R. Fundamentos em Gestão de Projetos - Construindo Competências para Gerenciar Projetos. 3. ed. São Paulo: Atlas, 2011.

CHAVES, M. S.; ARAÚJO, C.; TEIXEIRA, L. R.; GLÓRIA JÚNIOR, I.; ROSA, D.; NOGUEIRA, C. D. A New Approach to Managing Lessons Learned in the PMBoK Process Groups: The Ballistic 2.0 Model. International Journal of Information Systems and Project Management, v. 4, n. 1, p. 27-45, $2016 \mathrm{a}$.

CHAVES, M. S.; TESSI, M.; WINTER, R.; DAMASCENO JR., J. Validating a Collaborative Lessons Learned Model: A Multiple Cases Analysis. In: XL Encontro da ANPAD - EnANPAD, Costa do Sauípe/BA. Anais... Rio de Janeiro: ANPAD, 2016b.

DUFFIELD, S.; WHITTY, S. J. Developing a systemic lessons learned knowledge model for organisational learning through projects. International Journal of Project Management, v. 33, n. 2, p. 311-324, 2015.

GHOLAMI, B.; MURUGESAN, S. Global IT Project Management Using Web 2.0. International Journal of Information Technology Project Management, v. 2, n. 3, p. 30-52, 2011. http://doi.org/10.4018/jitpm.2011070103

GOMES. Avaliação do desempenho de processos. 2012. Disponível em: <https://bpmquotes.wordpress.com/2012/01/21/10-avaliacao-de-desempenho-de-processos-monitoramento/>. 
Acesso em: 20/05/2015.

GRACE, T. P. L. Wikis as a knowledge management tool. Journal of Knowledge Management, v. 13, n. 4, p. 6474, 2009. http://doi.org/10.1108/13673270910971833

HEVNER, A. R. A three cycle view of design science research. Scandinavian journal of information systems, v. 19, n. 2, p. 4, 2007.

KERZNER, H. Gerenciamento de Projetos - Uma abordagem sistêmica para planejamento, programação e controle. 10 ed. São Paulo: Blucher, 2011.

LEVY, M. WEB 2.0 implications on knowledge management. Journal of Knowledge Management, v. 13, n. 1, p. 120-134, 2009. http://doi.org/10.1108/13673270910931215

LINDNER, F.; WALD, A. Success factors of knowledge management in temporary organizations. International Journal of Project Management, v. 29, n. 7, p. 877-888, 2011. http://doi.org/10.1016/j.jproman.2010.09.003

MAJCHRZAK, A.; WAGNER, C.; YATES, D. Corporate wiki users: results of a survey. Proceedings of the 2006 International Symposium on Wikis WikiSym, v. 6, p. 99-104, 2006. http://doi.org/10.1145/1149453.1149472

MAXIMIANO, A. Administração de Projetos: Como transformar ideias em resultados. 2. ed. São Paulo: Atlas, 2002.

MELLO. Gerenciando 0 desempenho de processos de negócio, 2014. Disponível em: $<$ <ttp://blog.iprocess.com.br/2014/06/gerenciando-o-desempenho-de-processos/>. Acesso em: 22/05/2015.

MELOCHE, J.; HASAN, H.; WILLIS, D.; PFAFF, C.; QI, Y. (2009). Co-creating corporate knowledge with a wiki, 2009. Disponivel em: < http://ro.uow.edu.au/commpapers/673/>. Acesso em: 22/05/2015.

MILTON, N. The Lessons Learned Handbook: Practical approaches to learning from experience. Elsevier, 2010.

NATO. The NATO Lessons Learned Handbook, 2011. Disponivel em: <http://www.jallc.nato.int/newsmedia/docs/lessons_learned_handbook_2nd_edition.pdf>. Acesso em: 22/05/2015.

OLIVEIRA FILHO, J. A. Gestão de Projetos no Setor Público. Editorial deste Caderno de Estudos e Pesquisa, 2012.

PACHLER, N.; DALY, C. Narrative and learning with Web 2.0 technologies: towards a research agenda. Journal of Computer Assisted Learning, v. 25, n. 1, p. 6-18, 2009. http://doi.org/10.1111/j.1365-2729.2008.00303.x

PAPADOPOULOS, T.; STAMATI, T.; NOPPARUCH, P. Exploring the determinants of knowledge sharing via employee weblogs. International Journal of Information Management, v. 33, p. 133-146, 2013. http://doi.org/10.1016/j.jijnfomgt.2012.08.002

PEMSEL, S.; WIEWIORA, A. Project management office a knowledge broker in project-based organisations. International Journal of Project Management, v. 31, p. 31-42, 2013. http://doi.org/10.1016/j.ijproman.2012.03.004

PMI. Project Management Body Of Knowledge - PMBoK. 5. ed. São Paulo: Saraiva, 2013

REIS, G. P. Gestão de Projetos Complexos de Longo Prazo na Segurança Pública. Academia da Polícia Militar de MG, 2009.

RIEMER, K.; SCIFLEET, P. Enterprise Social Networking in Knowledge - intensive Work Practices : A Case Study in a Professional Service Firm, p. 1-12, 2012.

ROSA, D. V.; CHAVES, M. S.; OLIVEIRA, M.; PEDRON, C. Target: A Collaborative Model based on Social Media to Support the Management of Lessons Learned in Projects. International Journal of Managing Projects in Business, v. 9, n. 3, p. 1-30, 2016.

SCHINDLER, M.; EPPLER, M. J. Harvesting project knowledge: A review of project learning methods and success factors. International Journal of Project Management, v. 21, p. 219-228, 2003. http://doi.org/10.1016/S02637863(02)00096-0

STANDING, C.; KINITI, S. How can organizations use wikis for innovation? Technovation, v. 31, n. 7, p. 287-295, 2011. http://doi.org/10.1016/j.technovation.2011.02.005

STOCKER, A.; MÜLLER, J. Exploring Factual and Perceived Use and Benefits of a Web 2 . 0-based Knowledge

Revista Alcance - Eletrônica - vol. 24 - n. 1 - jan./mar. 2017 
Management Application: The Siemens Case References. International Conference on Knowledge Management and Knowledge Technologies, 2013. http://doi.org/10.1145/2494188.2494204

STOCKER, A.; RICHTER, A.; HOEFLER, P.; TOCHTERMANN, K. Exploring appropriation of enterprise wikis: A multiple-case study. Computer Supported Cooperative Work, v. 21, p. 317-356, 2012. http://doi.org/10.1007/s10606-012-9159-1

SVEJVIG, P.; ANDERSEN, P. Rethinking project management: A structured literature review with a critical look at the brave new world. International Journal of Project Management, v. 33, n. 2, p. 278-290, 2014. http://doi.org/10.1016/j.ijproman.2014.06.004

TURBAN, E.; LIANG, T. P.; WU, S. P. J. A Framework for Adopting Collaboration 2.0 Tools for Virtual Group Decision Making. Group Decision and Negotiation, v. 20, n. 2, p. 137-154, 2011. http://doi.org/10.1007/s10726010-9215-5

VERONESE, G. T.; CHAVES, M. An Integrated Approach to Collaborative Learning in Projects. Strategic Integration of Social Media into Project Management Practice, IGI Global, p. 160-178, 2016.

Verzuh, E. The fast forward in MBA in project management. New York, Chichester: J. Wiley and Sons, 1999.

VIROLAINEN, T. Learning from projects: a qualitative metasummary, School of Industrial Engineering and Management. Lappeenranta University of Technology, 2014

VON KROGH, G. How does social software change knowledge management? Toward a strategic research agenda. The Journal of Strategic Information Systems. v. 21, n. 2, p. 154-164, june 2012. http://doi.org/10.1016/j.jsis.2012.04.003

WEBER, R.; AHA, D. W.; BECERRA-FERNANDEZ, I. Intelligent lessons learned systems. Expert Systems with Applications, v. 20, p. 17-34, 2001. http://doi.org/10.1016/S0957-4174(00)00046-4

WIERINGA, R.; MORALI, A. Technical action research as a validation method in information systems design science. In: International Conference on Design Science Research in Information Systems, Springer Berlin Heidelberg, 2012. p. 220-238.

WIEWIORA, A.; MURPHY, G. Unpacking "lessons learned": investigating failures and considering alternative solutions. Knowledge Management Research \& Practice, v. 13, p. 17-30, 2013. http://doi.org/10.1057/kmrp.2013.26

WINTER, R.; CHAVES, M. S. Innovation in the management of lessons learned in an IT project with the adoption of social media. International Journal of Innovation, v. 5, n. 2, 2017.

YOON, C.; KIM, S. Convenience and TAM in a ubiquitous computing environment: The case of wireless LAN. Electronic Commerce Research and Applications, v. 6, p. 102-112, 2007. http://doi.org/10.1016/j.elerap.2006.06.009 